\title{
ON TOPOLOGICAL INVARIANTS ASSOCIATED WITH A POLYNOMIAL WITH ISOLATED CRITICAL POINTS
}

\author{
NICOLAS DUTERTRE \\ Université de Provence, Centre de Mathématiques et Informatique, 39 rue Joliot-Curie, \\ 13453 Marseille Cedex 13, France \\ e-mail:dutertre@cmi.univ-mrs.fr
}

(Received 30 April, 2003; accepted 7 January, 2004)

\begin{abstract}
We consider a polynomial $f: \mathbb{R}^{n} \rightarrow \mathbb{R}$ with isolated critical points and we relate $\chi\left(f^{-1}(0)\right)$ and $\chi(\{f \geq 0\})-\chi(\{f \leq 0\})$ to the topological degrees of polynomial maps defined in terms of $f$.
\end{abstract}

2000 Mathematics Subject Classification. 14P25.

1. Introduction. Let $F=\left(F_{1}, \ldots, F_{k}\right): \mathbb{R}^{n} \rightarrow \mathbb{R}^{k}$ be a polynomial mapping and let $W=F^{-1}(0)$. Let $G_{1}, \ldots, G_{l}$ be polynomials. An interesting problem is the computation of $\chi(W)$ and $\chi\left(W \cap\left\{G_{1} \geq 0, \ldots, G_{l} \geq 0\right\}\right)$ in terms of the polynomials $F_{i}$ and $G_{j}$.

When $W$ is compact, Szafraniec [17] and Bruce [4] proved that there exists a polynomial $P: \mathbb{R}^{n+1} \rightarrow \mathbb{R}$ with an algebraically isolated critical point at the origin such that

$$
\chi(W)=\frac{1}{2}\left((-1)^{n}-\operatorname{deg}_{0} \nabla P\right)
$$

where $\operatorname{deg}_{0} \nabla P$ is the topological degree at the origin of the gradient of $P$. The study of the case of $W$ non-compact has been done in $[6,18,19]$, but only when $1 \leq k<n$ and $W$ is a smooth manifold of dimension $n-k$. In [18], Szafraniec constructs a polynomial map $H: \mathbb{R}^{n+k} \rightarrow \mathbb{R}^{n+k}$. He proves that $H^{-1}(0) \subset B_{R}^{n+k}$, where $B_{R}^{n+k}$ is a ball in $\mathbb{R}^{n+k}$ centered at the origin with sufficiently big radius $R$, and that $\chi(W)=(-1)^{k} \operatorname{deg} h$, where $h=H /\|H\|: S_{R}^{n+k-1} \rightarrow S^{n+k-1}$ and $S_{R}^{n+k-1}=\partial B_{R}^{n+k}$. In [6], the authors consider a polynomial algebra $A$ and they prove, assuming $\operatorname{dim}_{\mathbb{R}} A<+\infty$, that

$$
\chi(W) \equiv \operatorname{dim}_{\mathbb{R}} A \bmod 2 .
$$

This latter formula is refined in [19], where it is proved that there exist two bilinear symmetric forms $\Phi$ and $\Phi_{M}$ on $A$ such that

$$
\begin{aligned}
& \text { if } n-k \text { is odd } \chi(W)=(-1)^{k} \text { signature } \Phi, \\
& \text { if } n-k \text { is even } \chi(W)=\text { signature } \Phi_{M} .
\end{aligned}
$$

In [8], we started the investigation of the case in which $W$ admits a finite number of singularities. We generalize first formula (1) above and we obtain

$$
\chi(W)+\Sigma_{\mu} \equiv \operatorname{dim}_{\mathbb{R}} A \bmod 2,
$$


where $\Sigma_{\mu}$ is the sum of the Milnor numbers at the singularities of $W$. Then we generalize formulae (2) but only in the cases of curves $(k=n-1)$ and of odd-dimensional hypersurfaces ( $k=1$ and $n$ is even).

The first aim of this paper is to solve the case of even-dimensional hypersurfaces with isolated singularities. Actually we give a new method that works for both parities. We consider a polynomial $f: \mathbb{R}^{n} \rightarrow \mathbb{R}$ with a finite number of critical points, some of them possibly lying in the fibre $f^{-1}(0)$. We make the additional assumption that $f(0)>0$. Taking $(x, \lambda)=\left(x_{1}, \ldots, x_{n}, \lambda\right)$ as a coordinate system for $\mathbb{R}^{n+1}$, we define four polynomial mappings $H, K, L_{1}$ and $L_{2}$ in the following way : $H(x, \lambda)=(\lambda x+\nabla f, f)$, $K(x, \lambda)=(\lambda x+\nabla f, \lambda f), L_{1}(x, \lambda)=(\nabla f, \lambda f-1)$ and $L_{2}(x, \lambda)=\left(\nabla f, \lambda f^{2}-1\right)$. Here $\nabla f$ denotes the gradient vector of $f$. We prove, in our Theorem 5.10, that the zero sets of these applications are compact and that, if $n$ is even, then

$$
\begin{aligned}
\chi\left(f^{-1}(0)\right) & =\operatorname{deg} H+\operatorname{deg} \nabla f-\operatorname{deg} L_{2}, \\
\chi(\{f \geq 0\})-\chi(\{f \leq 0\}) & =1-\operatorname{deg} K-\operatorname{deg} L_{1},
\end{aligned}
$$

and if $n$ is odd, then

$$
\begin{aligned}
\chi\left(f^{-1}(0)\right) & =\operatorname{deg} K-\operatorname{deg} L_{1}, \\
\chi(\{f \geq 0\})-\chi(\{f \leq 0\}) & =1-\operatorname{deg} H-\operatorname{deg} \nabla f+\operatorname{deg} L_{2} .
\end{aligned}
$$

By deg $H$, which we call the total degree of $H$, we mean the topological degree of the $\operatorname{map} \frac{H}{\|H\|}: S_{R^{\prime}}^{n} \rightarrow S^{n}$, where $S_{R^{\prime}}^{n}=\partial B_{R^{\prime}}^{n+1}$ and $H^{-1}(0) \subsetneq B_{R^{\prime}}^{n+1}$.

These formulae are global polynomial versions of a result due to Khimshiasvili on the Euler characteristic of the real Milnor fibre. It states that, if $g:\left(\mathbb{R}^{n}, 0\right) \rightarrow(\mathbb{R}, 0)$ is an analytic function-germ with an isolated critical point at the origin, then

$$
\chi\left(g^{-1}(\delta) \cap B_{\varepsilon}^{n}\right)=1-\operatorname{sign}(-\delta)^{n} \operatorname{deg}_{0} \nabla g,
$$

for any regular value $\delta$ of $g, 0<|\delta| \ll \varepsilon \ll 1$. Here $\operatorname{deg}_{0} \nabla g$ is the topological degree of $\frac{\nabla g}{\|\nabla g\|}: S_{\varepsilon}^{n-1} \rightarrow S^{n-1}$. A proof of this can be found in [1], [10], [14] or [21].

The proof of our main theorem is based on Morse theory for manifolds with corners. Putting $\omega(x)=\frac{1}{2}\left(x_{1}^{2}+\cdots+x_{n}^{2}\right)$, we study the critical points of Morse perturbations of $\omega_{\mid f-1(\delta) \cap B_{R}^{n}}, \omega_{\mid\{f \geq \delta\} \cap B_{R}^{n}}$ and $\omega_{\mid\{f \leq \delta\} \cap B_{R}^{n}}$, where $\delta$ is a regular value of $f$ close to 0 . These critical points are in bijection with non-degenerate zeros of $\tilde{H}_{\delta}$ and $\tilde{K}_{\delta}$, two appropriate perturbations of $H$ and $K$, and their Morse indices are related to the local degree of $\tilde{H}_{\delta}$ and $\tilde{K}_{\delta}$ at those zeros. This gives a link between $\chi\left(f^{-1}(\delta) \cap B_{R}^{n}\right)$ and $\chi\left(\{f \geq \delta\} \cap B_{R}^{n}\right)-\chi\left(\{f \leq \delta\} \cap B_{R}^{n}\right)$ and the topological degrees of $H$ and $K$. Then we relate $\chi\left(f^{-1}(0)\right)$ (respectively $\left.\chi(\{f \geq 0\})-\chi(\{f \leq 0\})\right)$ to $\chi\left(f^{-1}(\delta) \cap B_{R}^{n}\right)$ (respectively $\left.\chi\left(\{f \geq \delta\} \cap B_{R}^{n}\right)-\chi\left(\{f \leq \delta\} \cap B_{R}^{n}\right)\right)$.

In Section 2, we recall some facts about Morse theory for manifolds with corners. In Section 3, we give methods for the computation of the total degree of a polynomial mapping. These methods will be useful in the application of our theorems to concrete examples. Section 4 is devoted to some technical lemmas : we relate a Morse index to a local topological degree. Finally we prove our degree formulae in Section 5.

Some computations are given at the end of the paper. They have been done with a program written by Andrzej Lecki. The author is very grateful to him and Zbigniew Szafraniec for giving him this program. 
This research has been supported by a Marie Curie Fellowship of The European Community programme Improving Human Research Potential and the Socio-economic Knowledge Base under contract number HPMFCT-2000-00645. It was done at the Centre de Recerca Matemàtica in Barcelona. The author wishes to thank this institution for its hospitality.

2. Morse theory for manifolds with corners. We generalize the notion of correct critical points and Morse correct functions, defined for manifolds with boundary in [13], to the case of manifolds with corners. Then we relate the Euler characteristic of a manifold with corners to the indices of correct critical points.

Let us start with some basic facts on manifolds with corners. Our reference is [5]. A manifold with corners $M$ is defined by an atlas of charts modelled on open subsets of $\mathbb{R}_{+}^{n}$. We write $\partial M$ for its boundary. We shall make the additional assumption that the boundary is partitioned into pieces $\partial_{i} M$, themselves manifolds with corners, such that in each chart, the intersections with the coordinate hyperplanes $x_{j}=0$ correspond to distinct pieces $\partial_{i} M$ of the boundary. For any set $I$ of suffices, we write $\partial_{I} M=\cap_{i \in I} \partial_{i} M$ and we make the convention that $\partial_{\emptyset} M=M \backslash \partial M$.

Any $n$-manifold $M$ with corners can be embedded in an $n$-manifold $M^{+}$without boundary so that the pieces $\partial_{i} M$ extend to submanifolds $\partial_{i} M^{+}$of codimension 1 in $M^{+}$. We shall assume that $M^{+}$is provided with a Riemannian metric.

Let $M$ be a manifold with corners and $\omega: M^{+} \rightarrow \mathbb{R}$ a smooth map. We consider the points $P$ that are critical points of $\omega_{\mid \partial_{I} M^{+}}$.

Definition 2.1. A critical point $P$ is correct (respectively Morse correct) if, taking $I(P):=\left\{i \mid P \in \partial_{i} M\right\}, P$ is a critical (respectively Morse critical) point of $\omega_{\mid \partial_{I(P)} M^{+}}$, and is not a critical point of $\omega_{\mid \partial_{J} M^{+}}$for any proper subset $J$ of $I(P)$.

Note that a 0 -dimensional corner point $P$ is always a critical point because in this case $\partial_{I(P)} M^{+}=\{P\}$, which is a 0 -dimensional manifold.

DEFINITION 2.2. The maps $\omega$ with all critical points Morse correct are called Morse correct.

Proposition 2.3. The set of Morse correct functions is dense and open in the space of all maps $M^{+} \rightarrow \mathbb{R}$.

Proof. This is clear from classical Morse theory, because there is a finite number of pieces $\partial_{I} M^{+}$.

The index $\lambda(P)$ of $\omega$ at a Morse correct point $P$ is defined to be that of $\omega_{\mid \partial_{I(P)} M^{+}}$. If $P$ is a correct critical point of $\omega, i \in I(P)$, and $J$ is formed from $I(P)$ by deleting $i$, then in a chart at $P$ with $\partial_{J} M$ mapping to $\mathbb{R}_{+}^{p}$ and $\partial_{I(P)} M$ to the subset $x_{1}=0$, the function $\omega$ is non-critical, but its restriction to $x_{1}=0$ is. Hence $\partial \omega / \partial x_{1} \neq 0$.

Definition 2.4. We say that $\omega$ is inward at $P$ if, for each $i \in I(P)$, we have $\partial \omega / \partial x_{1}>0$.

REMARK 2.5. By our convention, if $I(P)=\emptyset$, then $\omega$ is inward at $P$.

THEOREM 2.6. If $M$ is compact and $\omega$ is Morse correct, then

$$
\chi(M)=\sum\left\{(-1)^{\lambda(P)} \mid P \text { is an inward Morse critical point }\right\} .
$$


Proof. This is a consequence of stratified Morse theory [11, 12]. A good summary of the results we use can be found in [3, Section 2].

The manifold with corners $M$ is a compact Whitney stratified set of $M^{+}$, with stratum the $\partial_{I} M$. The function $\omega: M \rightarrow \mathbb{R}$ is easily seen to be a Morse function in the sense of [11] and so

$$
\chi(M)=\sum\{\alpha(\omega, P) \mid P \text { correct critical point }\},
$$

where

$$
\alpha(\omega, P)=1-\chi\left(\omega^{-1}(\omega(P)-\delta) \cap B(P, \varepsilon)\right),
$$

with $0<\delta \ll \varepsilon \ll 1$. Here $B(P, \varepsilon)$ is the ball centered at $P$ of radius $\varepsilon$ in the Riemannian manifold $M^{+}$. If $P$ belongs to $\partial_{\emptyset} M$ then $\alpha(\omega, P)$ is exactly $(-1)^{\lambda(P)}$. If $P$ belongs to $\partial_{I} M$, $I \neq \emptyset$, then $\alpha(\omega, P)=(-1)^{\lambda(P)} \cdot \alpha_{\text {nor }}(\omega, P)$, where $\alpha_{\text {nor }}(\omega, P)$ is the normal index of $\omega$ at $P$. It is defined as follows. Choose a normal slice $V$ at $P$; that is, a closed submanifold of $M^{+}$of dimension $n-\operatorname{dim} \partial_{I} M$, that intersects $\partial_{I} M$ in $P$ orthogonally. We obtain

$$
\alpha_{\text {nor }}(\omega, P)=1-\chi\left(\omega^{-1}(\omega(P)-\delta) \cap B(P, \varepsilon) \cap V\right) .
$$

Let us compute this normal index. We can assume that $\omega(P)=0$. Also we can choose a local chart $\left(x_{1}, \ldots, x_{n}\right)$ centered at $P$ such that $\partial_{I} M$ is given by $\left\{x_{1}=\ldots=x_{k}=0\right\}$ and $V$ is given by $\left\{x_{k+1}=\ldots=x_{n}=0\right\}, k<n$. Locally $M$ is the set $\left\{x_{1} \geq 0, \ldots, x_{k} \geq 0\right\}$. Furthermore, since $P$ is a correct point, $\partial \omega / \partial x_{j}(P) \neq 0$ for each $j \in\{1, \ldots, k\}$ and, by an appropriate change of coordinates, the restriction of $\omega$ to $V$ is just the linear form

$$
\sum_{j=1}^{k} \frac{\partial \omega}{\partial x_{j}}(P) x_{j} .
$$

It is then straightforward to see that $\alpha_{n o r}(\omega, P)=1$ if $\partial \omega / \partial x_{j}(P)>0$, for all $j \in$ $\{1, \ldots, k\}$, and $\alpha_{\text {nor }}(\omega, P)=0$ otherwise. This proves the theorem.

3. Total degree of a polynomial mapping. We study the topological degree on a big sphere of a polynomial mapping. Let $\left(x_{1}, \ldots, x_{N}\right)$ be a coordinate system in $\mathbb{R}^{N}$. Let $F=\left(F_{1}, \ldots, F_{N}\right): \mathbb{R}^{N} \rightarrow \mathbb{R}^{N}$ be a polynomial mapping such that $F^{-1}(0)$ is compact. There is $R \gg 0$ such that $F^{-1}(0) \subsetneq B_{R}^{N}$. Recall that deg $F$ stands for the topological degree of $\frac{F}{\|F\|}: S_{R}^{N-1} \rightarrow S^{N-1}$. We give two methods due to Szafraniec for computing $\operatorname{deg} H$. The first one [18] enables us to reduce this computation to the computation of a local degree at the origin. Let $I: \mathbb{R}^{N} \backslash\{0\} \rightarrow \mathbb{R}^{N} \backslash\{0\}$ be the inversion defined by $I(x)=x /\left\|x^{2}\right\|$, let $d_{i}$ denote the degree of the polynomial $F_{i}$ for each $i \in\{1, \ldots, N\}$ and let

$$
F^{\prime}(x)=\left(\|x\|^{2 d_{1}} \cdot F_{1} \circ I(x), \ldots,\|x\|^{2 d_{N}} \cdot F_{N} \circ I(x)\right) \text { for } x \neq 0 .
$$

Then $F^{\prime}$ can be extended to a polynomial map $\mathbb{R}^{N} \rightarrow \mathbb{R}^{N}$ such that 0 is isolated in $F^{\prime-1}(0)$. Let $r=1 / R$; the map

$$
\begin{aligned}
S_{r}^{N-1} & \rightarrow S_{R}^{N-1} \\
x & \mapsto I(x)
\end{aligned}
$$


is of degree +1. Clearly, the maps $F^{\prime}: S_{r} \rightarrow \mathbb{R}^{N} \backslash\{0\}$ and $F \circ I: S_{r} \rightarrow \mathbb{R}^{N} \backslash\{0\}$ are homotopic, and so, if $r$ is small and if $\operatorname{deg}_{0} F^{\prime}$ is the degree of $\frac{F^{\prime}}{\left\|F^{\prime}\right\|}$ around $S_{r}^{N-1}$, then $\operatorname{deg} F=\operatorname{deg}_{0} F^{\prime}$.

LEMMA 3.1. $\operatorname{deg} F=\operatorname{deg}_{0} F^{\prime}$.

Using the Eisenbud-Levine-Khimshiashvili's formula [9, 14], the computation of deg $H$ reduces to the problem of calculating a signature of an appropriate bilinear symmetric form. Unfortunately the formula of the above lemma is difficult to implement because it involves polynomials with a large number of monomials. However, if we add the assumption that the polynomial factor algebra $A_{F}=\frac{\mathbb{R}\left[x_{1}, \ldots, x_{N}\right]}{\left(F_{1}, \ldots, F_{N}\right)}$ is finite dimensional as a vector space over $\mathbb{R}$, then we can use the following more effective method. Let $\phi: A_{F} \rightarrow \mathbb{R}$ be the Kronecker symbol or global residue on $A_{F}$. A description of this residue can be found in $[2,7, \mathbf{1 6}, \mathbf{1 9}, 20]$. It is a linear functional with which we can define the following bilinear symmetric form $\Phi$ :

$$
\Phi: A_{F} \times A_{F} \rightarrow \mathbb{R}, \Phi(f, g)=\phi(f g) .
$$

THEOREM 3.2. The form $\Phi$ is non-degenerate and

$$
\operatorname{deg} F=\text { signature } \Phi .
$$

Proof. See [20, Theorem 1.5].

Now we assume that $F^{-1}(0)$ is a finite set, which is realized if $\operatorname{dim}_{\mathbb{R}} A_{F}<+\infty$. Let $q_{1}, \ldots, q_{t}$ be the zeros of $F$ and for all $i \in\{1, \ldots, t\}$, let $\operatorname{deg}_{q_{i}} F$ be the degree of $\frac{F}{\|F\|}$ around a small sphere centered at $q_{i}$. Let $P: \mathbb{R}^{N} \rightarrow \mathbb{R}$ be a polynomial. We wish to compute

$$
\sum_{i=1}^{t} \operatorname{sign} P\left(q_{i}\right) \cdot \operatorname{deg}_{q_{i}} F
$$

We write $(x, \lambda)=\left(x_{1}, \ldots, x_{n}, \lambda\right)$ for a coordinate system in $\mathbb{R}^{N+1}$ and we define $G$ : $\mathbb{R}^{N+1} \rightarrow \mathbb{R}^{N+1}$ by $G(x, \lambda)=(F, \lambda P-1)$.

LEMMA 3.3. The set $G^{-1}(0)$ is finite and

$$
\sum_{i} \operatorname{sign} P\left(q_{i}\right) \cdot \operatorname{deg}_{q_{i}} F=\operatorname{deg} G
$$

Proof. A point $(x, \lambda)$ belongs to $G^{-1}(0)$ if and only if $F(x)=0$ and $P(x) \neq 0$. Hence

$$
G^{-1}(0)=\left\{\left(q_{i}, \frac{1}{P\left(q_{i}\right)}\right) \mid P\left(q_{i}\right) \neq 0\right\}
$$

and

$$
\operatorname{deg} G=\sum_{i \mid P\left(q_{i}\right) \neq 0} \operatorname{deg}_{\left(q_{i}, \frac{1}{P(q i)}\right)} G .
$$

Changing $F$ if necessary, we can assume that $q_{i}$ is a non-degenerate zero of $F$. It is then a simple determinant computation to see that $\left(q_{i}, \frac{1}{P\left(q_{i}\right)}\right)$ is a non-degenerate zero of $G$ 
and that

$$
\operatorname{deg}_{\left(q_{i}, \frac{1}{P(q i)}\right)} G=\operatorname{sign} P\left(q_{i}\right) \cdot \operatorname{deg}_{q_{i}} F .
$$

4. An index computation. We characterize a Morse correct critical point of an analytic function defined on an analytic manifold with boundary. We relate its Morse index to a local topological degree.

Let $f: \mathbb{R}^{n} \rightarrow \mathbb{R}$ be an analytic function and let $p \in f^{-1}(0)$ be such that $\nabla f(p) \neq$ 0 . From the implicit function theorem, $f^{-1}(0)$ is a smooth $(n-1)$-manifold in the neighborhood of $p$. Let $\omega:\left(\mathbb{R}^{n}, p\right) \rightarrow(\mathbb{R}, \omega(p))$ be an analytic function defined around $p$. Let $H: \mathbb{R}^{n+1} \rightarrow \mathbb{R}^{n+1}$ be given by

$$
H(x, \lambda)=(\lambda \nabla \omega(x)+\nabla f(x), f(x)) .
$$

We shall study the situation at the point $p$.

LEMMA 4.1. The function $\omega_{\mid\{f * 0\}}(*$ is either $\leq$ or $\geq)$ admits a correct critical point at $p$ if and only if there exists $\lambda \neq 0$ such that $H(p, \lambda)=0$. Furthermore $\lambda$ is uniquely determined.

Proof. A point $p \in f^{-1}(0)$ is a critical point of $\omega_{\mid\{f * 0\}}$ if and only if there exists $\mu$ such that $\nabla \omega(p)+\mu \nabla f(p)=0$. Moreover it is correct if and only if $\mu \neq 0$. The number $\lambda$ sought is thus $1 / \mu$. If there is $\lambda^{\prime} \neq \lambda$ with $H\left(p, \lambda^{\prime}\right)=0$ then $\nabla \omega(p)=0$, which contradicts the fact that $p$ is correct.

LEMMA 4.2. The function $\omega_{\mid\{f * 0\}}$ admits a Morse correct critical point at $p$ if and only if there exists $\lambda \neq 0$ such that $H(p, \lambda)=0$ and $J H(p, \lambda) \neq 0, J H$ being the Jacobian determinant of $H$. Furthermore, if $s$ is the Morse index of $\omega_{f^{-1}(0)}$ at $p$ then

$$
(-1)^{s}=\operatorname{sign} \lambda^{n} \times \operatorname{sign} J H(p, \lambda) .
$$

Proof. Let $\bar{H}: \mathbb{R}^{n+1} \rightarrow \mathbb{R}^{n+1}$ be defined by

$$
\bar{H}(x, \lambda)=(\nabla \omega(x)+\lambda \nabla f(x), f(x)) .
$$

In [18], Szafraniec proves in Lemma 1.4 that $\omega_{\mid f^{-1}(0)}$ has a Morse critical point at $p$ if and only if there is a unique $\mu$ such that $\bar{H}(p, \mu)=0$ and $J \bar{H}(p, \mu) \neq 0$. In this case, $(-1)^{s+1}=\operatorname{sign} J \bar{H}(p, \mu)$. Now

$$
J \bar{H}(p, \mu)=\operatorname{det}\left(\bar{a}_{i, j}\right)_{1 \leq i, j \leq n+1},
$$

where

$$
\begin{aligned}
\bar{a}_{i, j} & =\frac{\partial^{2} \omega}{\partial x_{i} \partial x_{j}}(p)+\mu \frac{\partial^{2} f}{\partial x_{i} \partial x_{j}}(p) \quad \text { for }(i, j) \in\{1, \ldots, n\}^{2}, \\
\bar{a}_{i, n+1} & =\bar{a}_{n+1, i}=\frac{\partial f}{\partial x_{i}}(p) \quad \text { for } i \in\{1, \ldots, n\}, \\
\bar{a}_{n+1, n+1} & =0 .
\end{aligned}
$$

Then

$$
J \bar{H}(p, \mu)=\mu^{n-1} \times \operatorname{det}\left(a_{i, j}\right)_{1 \leq i, j \leq n+1},
$$


where

$$
a_{i, j}=\frac{1}{\mu} \frac{\partial^{2} \omega}{\partial x_{i} \partial x_{j}}(p)+\frac{\partial^{2} f}{\partial x_{i} \partial x_{j}}(p) \quad \text { for }(i, j) \in\{1, \ldots, n\}^{2},
$$

and $a_{i, j}=\bar{a}_{i, j}$ otherwise. Putting $\lambda=1 / \mu$ and using the fact that $-\lambda \frac{\partial \omega}{\partial x_{i}}(p)=\frac{\partial f}{\partial x_{i}}(p)$ for all $i \in\{1, \ldots, n\}$, we see that $J H(p, \lambda)=-\lambda^{n-2} J \bar{H}(p, \mu)$.

5. Degree formulas. Recall that $f: \mathbb{R}^{n} \rightarrow \mathbb{R}$ is a polynomial with isolated critical points and that $f(0)>0$. Let $\omega(x)=\frac{1}{2}\left(x_{1}^{2}+\cdots+x_{n}^{2}\right)$. The polynomials $H, K, L_{1}$ and $L_{2}$ are defined this way : $H(x, \lambda)=(\lambda x+\nabla f, f), K(x, \lambda)=(\lambda x+\nabla f, \lambda f), L_{1}(x, \lambda)=$ $(\nabla f, \lambda f-1)$ and $L_{2}(x, \lambda)=\left(\nabla f, \lambda f^{2}-1\right)$.

By Lemma 3.3, we already know that $L_{1}^{-1}(0)$ and $L_{2}^{-1}(0)$ are finite. We shall describe the set $H^{-1}(0)$ and $K^{-1}(0)$. We define $\Sigma_{f}:=\{\nabla f=0\}, \Sigma_{0}:=\Sigma_{f} \cap f^{-1}(0)$ and $M:=$ $f^{-1}(0) \backslash \Sigma_{0}$. It is clear that $M$ is either empty or a smooth manifold of dimension $n-1$. The polynomial function $\omega_{\mid M}$ has a finite number of critical values [15, Corollary 2.8] which implies that the set $C$ of critical points of $\omega_{\mid M}$ is bounded.

Lemma 5.1. A point $p$ belongs to $C$ if and only if there exists $\lambda \neq 0$ such that $H(p, \lambda)=0$. Furthermore $\lambda$ is uniquely determined.

Proof. Since $f(0)>0$, each critical point of $\omega_{\mid M}$ is a correct critical point. The lemma is a consequence of Lemma 4.1.

LemMA 5.2. A point p belongs to $\Sigma_{0}$ if and only if $H(p, 0)=0$.

Proof. This is clear.

Let $\Pi_{x}: \mathbb{R}^{n+1} \rightarrow \mathbb{R}^{n}$ be the projection on the $n$ first components.

Corollary 5.3. The set $\Pi_{x}\left(H^{-1}(0)\right)$ is $C \sqcup \Sigma_{0}$.

Proof. This follows from the two previous lemmas.

LEMMA 5.4. The set $H^{-1}(0)$ is compact.

Proof. We know that $\Pi_{x}\left(H^{-1}(0)\right)$ is bounded because $C$ is and $\Sigma_{0}$ is finite. Moreover it is closed because it is the algebraic set defined by the vanishing of $f$ and all the $2 \times 2$ minors of the jacobian matrix of the map $(f, \omega)$. Hence it is compact. For all $p \in \Pi_{x}\left(H^{-1}(0)\right)$, there exists a unique $\lambda(p)$ such that

$$
\lambda(p) \cdot p+\nabla f(p)=0 .
$$

Since $p \neq 0$, the map of $\Pi_{x}\left(H^{-1}(0)\right)$ given by $p \mapsto \lambda(p)$ is continuous and so $H^{-1}(0)=$ $\left\{(p, \lambda(p)) \mid p \in \Pi_{x}\left(H^{-1}(0)\right)\right\}$ is compact.

Lemma 5.5. A point $p$ belongs to $C$ if and only if there exists $\lambda \neq 0$ such that $K(p, \lambda)=0$. Furthermore $\lambda$ is uniquely determined .

LEMma 5.6. A point p belongs to $\Sigma_{f}$ if and only if $K(p, 0)=0$.

COROLlary 5.7. The set $\Pi_{x}\left(K^{-1}(0)\right)$ equals $C \sqcup \Sigma_{f}$.

LEMMA 5.8. The set $K^{-1}(0)$ is compact.

Proof. It is the union of $H^{-1}(0)$ and $\left\{(x, 0) \mid x \in \Sigma_{f}\right\}$. 
The expressions deg $H$ and $\operatorname{deg} K$ do make sense. We choose $R>0$ such that $C \cup \Sigma_{f} \subset B_{R}^{n}$. This implies that $f^{-1}(0) \cap B_{R}^{n}$ (respectively $\{f * 0\} \cap B_{R}^{n}, * \in\{\leq, \geq\}$ ) is a deformation retract of $f^{-1}(0)$ (respectively $\{f * 0\}$ ). Let us write $\Sigma_{f}=\left\{q_{1}, \ldots, q_{t}\right\}$ with $\Sigma_{0}=\left\{q_{1}, \ldots, q_{r}\right\}(r \leq t)$. We need the following lemma.

LEMMA 5.9. If $\delta$ is a small regular value of $f$, then

$$
\begin{aligned}
& \chi\left(f^{-1}(\delta) \cap B_{R}^{n}\right)=\chi\left(f^{-1}(0)\right)-\operatorname{sign}(-\delta)^{n} \sum_{i=1}^{r} \operatorname{deg}_{q_{i}} \nabla f, \\
& \chi\left(\{f \geq \delta\} \cap B_{R}^{n}\right)-\chi\left(\{f \leq \delta\} \cap B_{R}^{n}\right) \\
& \quad=\chi(\{f \geq 0\})-\chi(\{f \leq 0\})+\operatorname{sign}(-\delta)^{n+1} \sum_{i=1}^{r} \operatorname{deg}_{q_{i}} \nabla f .
\end{aligned}
$$

Proof. The first item is proved in exactly the same way as Khimshiasvili's formula mentioned in the introduction. We refer to $[\mathbf{1}, \mathbf{1 0}, \mathbf{1 4}, \mathbf{2 1}]$ for a proof.

In order to prove the second equation, for $\delta>0$, we use the facts that

$$
\chi\left(\{f \geq 0\} \cap B_{R}^{n}\right)=\chi\left(\{f \geq \delta\} \cap B_{R}^{n}\right)+\chi\left(\{0 \leq f \leq \delta\} \cap B_{R}^{n}\right)-\chi\left(f^{-1}(\delta) \cap B_{R}^{n}\right),
$$

and

$$
\chi\left(\{f \leq \delta\} \cap B_{R}^{n}\right)=\chi\left(\{f \leq 0\} \cap B_{R}^{n}\right)+\chi\left(\{0 \leq f \leq \delta\} \cap B_{R}^{n}\right)-\chi\left(f^{-1}(0) \cap B_{R}^{n}\right),
$$

and that $\{0 \leq f \leq \delta\} \cap B_{R}^{n}$ retracts to $f^{-1}(0) \cap B_{R}^{n}$. Applying this to $-f$ gives the result for $\delta<0$.

THEOREM 5.10. If $n$ is even, then

$$
\begin{aligned}
\chi\left(f^{-1}(0)\right) & =\operatorname{deg} H+\operatorname{deg} \nabla f-\operatorname{deg} L_{2}, \\
\chi(\{f \geq 0\})-\chi(\{f \leq 0\}) & =1-\operatorname{deg} K-\operatorname{deg} L_{1} .
\end{aligned}
$$

If $n$ is odd, then

$$
\begin{aligned}
\chi\left(f^{-1}(0)\right) & =\operatorname{deg} K-\operatorname{deg} L_{1}, \\
\chi(\{f \geq 0\})-\chi(\{f \leq 0\}) & =1-\operatorname{deg} H-\operatorname{deg} \nabla f+\operatorname{deg} L_{2} .
\end{aligned}
$$

Proof. Let us choose $R^{\prime}>0$ such that $H^{-1}(0) \subsetneq B_{R^{\prime}}^{n+1}$ and $K^{-1}(0) \subsetneq B_{R^{\prime}}^{n+1}$. Since $\Pi_{x}\left(K^{-1}(0)\right) \subset C \cup \Sigma_{f}$, we can choose $R^{\prime} \geq R$. Let $\delta \neq 0$ be a small regular value of $f$. We construct two appropriate deformations $H_{\delta}$ and $K_{\delta}$ of $H$ and $K$ in the following way:

$$
\begin{aligned}
& H_{\delta}(x, \lambda)=(\lambda x+\nabla f(x), f(x)-\delta), \\
& K_{\delta}(x, \lambda)=(\lambda x+\nabla f(x), \lambda(f(x)-\delta)) .
\end{aligned}
$$

We study first the topological degree of $\frac{K_{\delta}}{\left\|K_{\delta}\right\|}$ around $S_{R^{\prime}}^{n}$. Let

$$
m=\min \left\{\|K(x, \lambda)\| \mid(x, \lambda) \in S_{R^{\prime}}^{n}\right\} .
$$

On $S_{R^{\prime}}^{n},\left\|K-K_{\delta}\right\|=\lambda \delta$ and, if we take $\delta$ such that $\left|\delta R^{\prime}\right|<\frac{m}{2}$, then $\left\|K_{\delta}\right\|>\frac{m}{2}$ on $S_{R^{\prime}}^{n}$. This implies that this degree is well defined. We denote it by $\operatorname{deg}\left(K_{\delta}, R^{\prime}\right)$. 
If there is a point $(p, \lambda) \in S_{R^{\prime}}^{n}$ such that $K(p, \lambda)$ and $K_{\delta}(p, \lambda)$ point in opposite directions, then $\lambda p+\nabla f(p)=0$, for $K$ and $K_{\delta}$ have the same $n$ first components. Hence $\lambda f(p)$ and $\lambda(f(p)-\delta)$ have opposite signs. This can happen only if $|f(p)|<|\delta|$. But in this case $\|K(p, \lambda)\|<\left|\delta R^{\prime}\right|<\frac{m}{2}$, a contradiction. We have proved that $\operatorname{deg}\left(K_{\delta}, R^{\prime}\right)=$ $\operatorname{deg} K$. Similarly, $\operatorname{deg}\left(H_{\delta}, R^{\prime}\right)=\operatorname{deg} H$.

Let $(p, \lambda) \in H_{\delta}^{-1}(0) \cap B_{R^{\prime}}^{n+1}$. By Lemma $4.1, p$ is a critical point of $\omega_{\mid f^{-1}(\delta)}$ and $\|p\| \leq R^{\prime}$. Since on $\left\{R \leq\|x\| \leq R^{\prime}\right\}, \omega_{\mid f^{-1}(0)}$ does not admit critical points, $\omega_{\mid f^{-1}(\delta)}$ does not admit critical points on $\left\{R \leq\|x\| \leq R^{\prime}\right\}$, for $\delta$ sufficiently small. Hence $\|p\| \leq R$. Conversely, if $p$ is a critical point of $\omega_{\mid f^{-1}(\delta) \cap B_{R}^{n}}$, then there exists $\lambda$ such that $H_{\delta}(p, \lambda)=0$. Taking $\delta$ small enough, $p$ is close to $C \cup \Sigma_{0}$ and so, by continuity, $(p, \lambda)$ is close to $H^{-1}(0)$. Hence $(p, \lambda) \in B_{R^{\prime}}^{n+1}$. We have proved that $\Pi_{x}\left(H_{\delta}^{-1}(0) \cap B_{R^{\prime}}^{n+1}\right)$ is exactly the set of critical points of $\omega_{\mid f^{-1}(\delta) \cap B_{R}^{n}}$ that we denote by $C_{\delta}$. Similarly $\Pi_{x}\left(K_{\delta}^{-1}(0) \cap B_{R^{\prime}}^{n+1}\right)=$ $C_{\delta} \sqcup \Sigma_{f}$.

Let us compute $\operatorname{deg}\left(H, R^{\prime}\right)$. We choose a function $\tilde{\omega}: \mathbb{R}^{n} \rightarrow \mathbb{R}$ that uniformly approximates $\omega$ in the Whitney $C^{2}$-topology and such that $\tilde{\omega}_{\mid f^{-1}(\delta) \cap B_{R}^{n}}$ is Morse correct. One notices that, since the gradient of $\omega$ is outward pointing along $f^{-1}(0) \cap S_{R}^{n-1}$, $\tilde{\omega}_{\mid f^{-1}(\delta) \cap B_{R}^{n}}$ is not inward at any critical point lying in $f^{-1}(\delta) \cap S_{R}^{n-1}$. Let $\left\{p_{1}, \ldots, p_{m}\right\}$ be the set of critical points of $\tilde{\omega}_{\mid f^{-1}(\delta) \cap B_{R}^{n}}$ lying in $\{\|x\|<R\}$ and let $\left\{s_{1}, \ldots, s_{m}\right\}$ be the set of their respective indices. Since $f(0)>0, \omega_{\mid\{f \geq \delta\}}$ and $\omega_{\mid\{f \leq \delta\}}$ are correct and so are $\tilde{\omega}_{\mid\{f \geq \delta\}}$ and $\tilde{\omega}_{\mid\{f \leq \delta\}}$.

By Lemma 4.1 , for all $j \in\{1, \ldots, m\}$ there exists $\lambda_{j} \neq 0$ such that $\lambda_{j} \nabla \tilde{\omega}\left(p_{j}\right)+$ $\nabla f\left(p_{j}\right)=0$. By Lemma 4.2, each $\left(p_{j}, \lambda_{j}\right)$ is a non-degenerate zero of $\tilde{H}_{\delta}$, that is defined by

$$
\tilde{H}_{\delta}(x, \lambda)=(\lambda \nabla \tilde{\omega}(x)+\nabla f(x), f-\delta),
$$

and

$$
(-1)^{s_{j}}=\operatorname{sign} \lambda_{j}^{n} \times \operatorname{sign} J \tilde{H}_{\delta}\left(p_{j}, \lambda_{j}\right)
$$

Summing over all the points $p_{j}$ and using the fact that $\tilde{H}_{\delta}$ is close to $H_{\delta}$, we obtain

$$
\operatorname{deg}\left(H_{\delta}, R^{\prime}\right)=\sum_{j=1}^{m} \operatorname{sign} \lambda_{j}^{n} \times(-1)^{s_{j}} .
$$

We have to compute $\operatorname{deg}\left(K_{\delta}, R^{\prime}\right)$. First we see that, putting

$$
\tilde{K}_{\delta}(x, \lambda)=(\lambda \nabla \tilde{\omega}(x)+\nabla f(x), \lambda(f-\delta)),
$$

the points $\left(p_{j}, \lambda_{j}\right)$ are non-degenerate zeros of $\tilde{K}_{\delta}$ and

$$
J \tilde{K}_{\delta}\left(p_{j}, \lambda_{j}\right)=\lambda_{j} J \tilde{H}_{\delta}\left(p_{j}, \lambda_{j}\right)
$$

Hence

$$
(-1)^{s_{j}}=\operatorname{sign} \lambda_{j}^{n-1} \times \operatorname{sign} J \tilde{K}_{\delta}\left(p_{j}, \lambda_{j}\right)
$$

The points $\left(q_{i}, 0\right)$ are the other zeros of $\tilde{K}_{\delta}$. Taking a Morse approximation of $f$ around a point $q_{i}$, if necessary, which gives us an approximation of $\tilde{K}_{\delta}$ near $\left(q_{i}, 0\right)$, we prove 
that

$$
\operatorname{deg}_{\left(q_{i}, 0\right)} \tilde{K}_{\delta}=\operatorname{sign}\left(f\left(q_{i}\right)-\delta\right) \times \operatorname{deg}_{q_{i}} \nabla f
$$

Finally we get that

$$
\operatorname{deg}\left(K_{\delta}, R^{\prime}\right)=\sum_{j=1}^{m} \operatorname{sign} \lambda_{j}^{n-1} \times(-1)^{s_{j}}+\sum_{i=1}^{t} \operatorname{sign}\left(f\left(q_{i}\right)-\delta\right) \times \operatorname{deg}_{q_{i}} \nabla f .
$$

Now we relate these two degrees to Euler characteristics. By Theorem 2.6, we have

$$
\begin{aligned}
\chi\left(f^{-1}(\delta) \cap B_{R}^{n}\right) & =\sum_{j=1}^{m}(-1)^{s_{j}}, \\
\chi\left(\{f \geq \delta\} \cap B_{R}^{n}\right) & =1+\sum_{j \mid \lambda_{j}<0}(-1)^{s_{j}}, \\
\chi\left(\{f \leq \delta\} \cap B_{R}^{n}\right) & =\sum_{j \mid \lambda_{j}>0}(-1)^{s_{j}} .
\end{aligned}
$$

The term 1 that appears in the second formula is the contribution of the point 0 , which

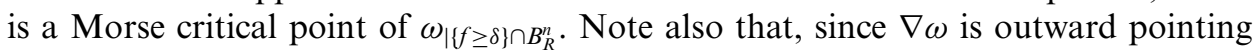
along $S_{R}^{n-1}$, no inward critical point lies on this sphere. From the two latter formulae, we deduce that

$$
\chi\left(\{f \geq \delta\} \cap B_{R}^{n}\right)-\chi\left(\{f \leq \delta\} \cap B_{R}^{n}\right)=1-\sum_{j=1}^{m} \operatorname{sign} \lambda_{j} \times(-1)^{s_{j}} .
$$

Collecting all this information, we have, if $n$ is even,

$$
\begin{gathered}
\chi\left(f^{-1}(\delta) \cap B_{R}^{n}\right)=\operatorname{deg} H \\
\chi\left(\{f \geq \delta\} \cap B_{R}^{n}\right)-\chi\left(\{f \leq \delta\} \cap B_{R}^{n}\right)-\sum_{i=1}^{t} \operatorname{sign}\left(f\left(q_{i}\right)-\delta\right) \cdot \operatorname{deg}_{q_{i}} \nabla f=1-\operatorname{deg} K .
\end{gathered}
$$

If $n$ is odd, then

$$
\begin{aligned}
& \chi\left(f^{-1}(\delta) \cap B_{R}^{n}\right)+\sum_{i=1}^{t} \operatorname{sign}\left(f\left(q_{i}\right)-\delta\right) \cdot \operatorname{deg}_{q_{i}} \nabla f=\operatorname{deg} K, \\
& \chi\left(\{f \geq \delta\} \cap B_{R}^{n}\right)-\chi\left(\{f \leq \delta\} \cap B_{R}^{n}\right)=1-\operatorname{deg} H .
\end{aligned}
$$

For $i \in\{1, \ldots, r\}, \operatorname{sign}\left(f\left(q_{i}\right)-\delta\right)=-\operatorname{sign}(\delta)$ and for $i \in\{r+1, \ldots, t\}$, we have $\operatorname{sign}\left(f\left(q_{i}\right)-\delta\right)=\operatorname{sign} f\left(q_{i}\right)$. Combining this with Lemma 5.9 yields, if $n$ is even,

$$
\begin{gathered}
\chi\left(f^{-1}(0)\right)-\sum_{i=1}^{r} \operatorname{deg}_{q_{i}} \nabla f=\operatorname{deg} H, \\
\chi(\{f \geq 0\})-\chi(\{f \leq 0\})-\sum_{i=r+1}^{t} \operatorname{sign}\left(f\left(q_{i}\right)\right) \cdot \operatorname{deg}_{q_{i}} \nabla f=1-\operatorname{deg} K .
\end{gathered}
$$


If $n$ is odd, then

$$
\begin{gathered}
\chi\left(f^{-1}(0)\right)+\sum_{i=r+1}^{t} \operatorname{sign}\left(f\left(q_{i}\right)\right) \cdot \operatorname{deg}_{q_{i}} \nabla f=\operatorname{deg} K, \\
\chi(\{f \geq 0\})-\chi(\{f \leq 0\})+\sum_{i=1}^{r} \operatorname{deg}_{q_{i}} \nabla f=1-\operatorname{deg} H .
\end{gathered}
$$

Finally, by Lemma 3.3,

$$
\sum_{i=1}^{r} \operatorname{deg}_{q_{i}} \nabla f=\operatorname{deg} \nabla f-\operatorname{deg} L_{2}
$$

and

$$
\sum_{i=r+1}^{t} \operatorname{sign} f\left(q_{i}\right) \cdot \operatorname{deg}_{q_{i}} \nabla f=\operatorname{deg} L_{1} .
$$

ExAMPLES. (1) Let $f\left(x_{1}, x_{2}\right)=-x_{1}^{2} x_{2}^{5}+x_{1}^{4} x_{2}^{3}+5 x_{2}^{3}-5 x_{1}^{2} x_{2}-4 x_{2}^{2}+4 x_{1}^{2}$. The computer gives that $\operatorname{dim} \mathbb{R}\left[x_{1}, x_{2}\right] /\left(f, f_{x_{1}}, f_{x_{2}}\right)=13$ so that $f^{-1}(0)$ may admit singularities. Let us consider $H$ and $K: \mathbb{R}^{3} \rightarrow \mathbb{R}^{3}$ given by

$$
\begin{aligned}
& H\left(x_{1}, x_{2}, x_{3}\right)=\left(x_{1} x_{3}+f_{x_{1}}, x_{3}\left(x_{2}-1\right)+f_{x_{2}}, f\right), \\
& K\left(x_{1}, x_{2}, x_{3}\right)=\left(x_{1} x_{3}+f_{x_{1}}, x_{3}\left(x_{2}-1\right)+f_{x_{2}}, f x_{3}\right) .
\end{aligned}
$$

Here we use the distance function $\omega\left(x_{1}, x_{2}\right)=\frac{1}{2}\left(x_{1}^{2}+\left(x_{2}-1\right)^{2}\right)$. Since $f(0,1)=1>0$, we can apply the previous theorems. Using methods of Section 3, we find $\operatorname{deg} H=5$, $\operatorname{deg} K=1, \operatorname{deg} \nabla f=-4, \operatorname{deg} L_{1}=-1$ and $\operatorname{deg} L_{2}=1$. By our theorem, we have

$$
\begin{gathered}
\chi\left(f^{-1}(0)\right)=5+(-4)-1=0, \\
\chi(\{f \geq 0\})-\chi(\{f \leq 0\})=1+(-1)+(-1)=-1 .
\end{gathered}
$$

(2) Let $f\left(x_{1}, x_{2}, x_{3}\right)=x_{1}^{2} x_{2}^{3}+x_{1} x_{2}^{4}-2 x_{1}^{3}-2 x_{1}^{2} x_{2}-x_{2}^{3}-x_{1} x_{2}+2 x_{1} x_{3}+x_{3}^{2}+$ $2 x_{1}+1$. First $\operatorname{dim} \mathbb{R}\left[x_{1}, x_{2}, x_{3}\right] /\left(f, f_{x_{1}}, f_{x_{2}}, f_{x_{3}}\right)=6$, so that $f^{-1}(0)$ may have singularities. We find that $\operatorname{deg} H=3, \operatorname{deg} K=5, \operatorname{deg} \nabla f=-2$ and $\operatorname{deg} L_{1}=\operatorname{deg} L_{2}=0$. Hence

$$
\begin{gathered}
\chi\left(f^{-1}(0)\right)=5-0=5, \\
\chi(\{f \geq 0\})-\chi(\{f \leq 0\})=1-3-(-2)=0 .
\end{gathered}
$$

REMARK 5.11. Formulas given in Theorem 5.10 are still true with the weaker hypothesis that the set of critical points of $f$ is compact and the proof is similar to the one we presented above. However, in that case, the maps $H, K, L_{1}$ and $L_{2}$ can not admit a finite number of zeros and so their total degrees are more difficult to compute. 


\section{REFERENCES}

1. V. I. Arnold, Index of a singular point of a vector field, the Petrovski-Oleinik inequality, and mixed Hodge structures, Funct. Anal. Appl. 12 (1978), 1-14.

2. E. Becker, J. P. Cardinal, M. F. Roy and Z. Szafraniec, Multivariate Bezoutians, Kronecker symbol and Eisenbud \& Levine formula, in Algorithms in algebraic geometry and applications, Progress in Mathematics 143 (Birkhauser, 1996), 79-104.

3. L. Brocker and M. Kuppe, Integral geometry of tame sets, Geometriae Dedicata $\mathbf{8 2}$ (2000), 285-323.

4. J. W. Bruce, Euler characteristics of real varieties, Bull. London Math. Soc. 22 (1990), $547-552$

5. J. Cerf, Topologie de certains espaces de plongements, Bull. Soc. Math. France 89 (1961), $227-380$

6. P. Dudzinski, A. Lecki, P. Nowak-Przygodzki and Z. Szafraniec, On the topological invariance of the Milnor number mod 2, Topology 32 (1993), 573-576.

7. N. Dutertre, An algebraic formula for the Euler characteristic of some semi-algebraic sets, J. Pure Appl. Algebra 139 (1999), 41-60.

8. N. Dutertre, On affine complete intersections with isolated singularities, J. Pure Appl. Algebra 146, No. 1-2 (2001), 129-147.

9. D. Eisenbud and H. I. Levine, An algebraic formula for the degree of a $C^{\infty}$ map-germ, Ann. of Math. 106 (1977), 19-44.

10. T. Fukui, Mapping degree formula for 2-parameter bifurcation of function-germs, Topology 32 (1993), 567-571.

11. M. Goresky and R. Mac Pherson, Stratified Morse theory (Springer-Verlag, 1988).

12. H. Hamm, On stratified Morse theory, Topology 38 (1999), 427-438.

13. H. Hamm and Tŕang, Lê Dũng, Un théorème de Zariski du type de Lefschetz, Ann. Sci. Ecol. Norm. Sup. (3) 6 (1973), 317-355.

14. G. M. Khimshiashvili, On the local degree of a smooth map, Soobshch. Akad. Nauk Gruz. SSR 85 (1977), 309-311.

15. J. Milnor, Singular points of complex hypersurfaces, Ann. Math. Stud. 61 (Princeton University Press, 1968).

16. G. Scheja and U. Storch, Über Spurfunktionen bei vollstandingen Durschnitten, J. Reine Angew Math. 278/279 (1975), 174-190.

17. Z. Szafraniec, On the Euler characteristic of analytic and algebraic sets, Topology 26 (1986), 411-414.

18. Z. Szafraniec, The Euler characteristic of algebraic complete intersections, J. Reine Angew Math. 397 (1989), 194-201.

19. Z. Szafraniec, A formula for the Euler characteristic of a real algebraic manifold, Manuscripta Math. 85 (1994), 345-360.

20. Z. Szafraniec, Topological degree and quadratic forms, J. Pure Appl. Algebra 141 (1999), 299-314.

21. C. T. C. Wall, Topological invariance of the Milnor number mod 2, Topology 22 (1983), $345-350$. 